Annales Geophysicae (2002) 20: 1049-1061 C European Geophysical Society 2002

\title{
Investigating the auroral electrojets with low altitude polar orbiting satellites
}

\author{
T. Moretto ${ }^{1}$, N. Olsen ${ }^{2}$, P. Ritter ${ }^{3}$, and G. Lu ${ }^{4}$ \\ ${ }^{1}$ Laboratory for Extraterrestrial Physics, NASA Goddard Space Flight Center, Greenbelt, Maryland, USA \\ ${ }^{2}$ Danish Space Research Institute, Copenhagen, Denmark \\ ${ }^{3}$ Geoforschungs-Zentrum Potsdam, Potsdam, Germany \\ ${ }^{4}$ High Altitude Observatory, Boulder, Colorado, USA
}

Received: 15 October 2001 - Revised: 16 April 2002 - Accepted: 17 May 2002

\begin{abstract}
Three geomagnetic satellite missions currently provide high precision magnetic field measurements from low altitude polar orbiting spacecraft. We demonstrate how these data can be used to determine the intensity and location of the horizontal currents that flow in the ionosphere, predominantly in the auroral electrojets. First, we examine the results during a recent geomagnetic storm. The currents derived from two satellites at different altitudes are in very good agreement, which verifies good stability of the method. Further, a very high degree of correlation (correlation coefficients of 0.8-0.9) is observed between the amplitudes of the derived currents and the commonly used auroral electrojet indices based on magnetic measurements at ground. This points to the potential of defining an auroral activity index based on the satellite observations, which could be useful for space weather monitoring. A specific advantage of the satellite observations over the ground-based magnetic measurements is their coverage of the Southern Hemisphere, as well as the Northern. We utilize this in an investigation of the ionospheric currents observed in both polar regions during a period of unusually steady interplanetary magnetic field with a large negative $Y$-component. A pronounced asymmetry is found between the currents in the two hemispheres, which indicates real inter-hemispheric differences beyond the mirrorasymmetry between hemispheres that earlier studies have revealed. The method is also applied to another event for which the combined measurements of the three satellites provide a comprehensive view of the current systems. The analysis hereof reveals some surprising results concerning the connection between solar wind driver and the resulting ionospheric currents. Specifically, preconditioning of the magnetosphere (history of the interplanetary magnetic field) is seen to play an important role, and in the winther hemisphere, it seems to be harder to drive currents on the nightside than on the dayside.
\end{abstract}

Key words. Ionosphere (electric fields and currents) -

Correspondence to: T. Moretto

(Therese.Moretto@gsfc.nasa.gov)
Magnetospheric physics (current systems; magnetosphereionosphere interactions)

\section{Introduction}

The currents flowing in the high latitude ionospheres constitute an important space weather parameter. They are a direct manifestation of the energy deposited in the near-Earth environment as a result of solar wind-magnetosphere interaction. In addition, they are a signature of the large-scale magnetospheric processes that drive them and, therefore, provide a measure for the state of the Earth's magnetosphereionosphere system. Last but not least, they are in themselves a cause of space weather effects, such as the generation of ground induced currents and increased drag on low altitude spacecraft through atmospheric heating. The most intense current system in the ionosphere is the auroral electrojets (AE). These flow in the auroral oval, the eastward auroral electrojet (EAEJ) in the dusk side of the oval and the westward auroral electrojet (WAEJ) in the dawn side. The WAEJ is occasionally fed by the closure of the substorm current wedge and hence, is where the most intense currents are found.

The ionospheric currents can be determined very reliably from ground-based magnetic measurements. This has been used successfully in a wealth of studies over the last thirty years to determine many features of the high-latitude ionospheric current systems as well as their relation to magnetospheric and solar wind drivers (Kamide et al., 1981; FriisChristensen et al., 1985; Kamide et al., 1994; Richmond et al., 1998, and many others).

As on ground, the effect of the horizontal ionospheric currents in the auroral electrojets are felt in the magnetic measurements made at low altitude, near-polar orbiting satellites. Thus such measurements offer an alternative means for determining and monitoring these currents. This was demonstrated with data from the MAGSAT satellite by Olsen (1996). In this study we explore the potential hereof further 
using the magnetic field measurements from three geomagnetic satellite missions: Ørsted, CHAMP, and the Ørsted-2 experiment on board the SAC-C satellite, all of which are currently in operation. Although the measurements are taken as far as $700 \mathrm{~km}$ from the source region of the currents in the ionosphere, the high precision and good stability of the measurements, together with an accurate and up-to-date internal geomagnetic field model, which these missions are designed to provide, make it possible to detect even the small ionospheric contribution.

In this paper, we first describe briefly the satellite measurements and outline the modelling method used to derive estimates of the ionospheric currents. The method is applied to Ørsted and CHAMP data from a recent geomagnetic storm period. This serves two purposes. First, we can verify that there is overall good agreement between the results from the two satellites. This provides a consistency check of the method. Second, we can verify that the level of current activity measured follows quite closely the activity level as estimated in the usual way from ground magnetometers. Both comparisons are also used to illustrate limitations of the method.

A period has been identified for which the two satellites cross along almost identical tracks and nearly simultaneously over opposite polar regions during a prolonged period of unusually steady solar wind conditions. This offers an unique opportunity to make a direct comparison of the electrojet currents in the Northern and Southern Hemispheres. We present the results of this comparison and interpret them in terms of the state of the solar wind-magnetosphere-ionosphere coupling, as determined by the value of the interplanetary magnetic field.

Finally, we show the results from another special sequence of orbits. In this case, the orbits of the three satellites are widely separated in local time and they all cross the same pole nearly simultaneously. Analyzing these results puts to the test our ability to relate observed ionospheric current systems in the two hemispheres to the value of the interplanetary magnetic field.

\section{Measuring the ionospheric currents from space}

In this section, we describe briefly the main characteristics of the satellite missions, their measurements, and the modeling technique that we apply to the data.

\subsection{The satellites and their orbits}

We use the high accuracy magnetic field measurements from the three satellites: Ørsted (Neubert et al., 2001), CHAMP (Lühr et al., 2001), and SAC-C. The CHAMP satellite is closest to the Earth in a near-circular orbit of altitude $450 \mathrm{~km}$. Ørsted is in a slightly elliptical orbit of $650-850 \mathrm{~km}$ altitude. The Ørsted-2 geomagnetic experiment is on board the SAC$\mathrm{C}$ satellite which is in a near-circular orbit of $700 \mathrm{~km}$ altitude. All three satellites are near-polar orbiting, but the drifts of their orbit planes in local time are different. SAC-C is in a sun-synchronous orbit at 10:00-22:00 LT; Ørsted's orbit is slowly drifting, by roughly $1 \mathrm{~h}$ of LT for every 2 months; and CHAMP's orbit drifts roughly $3 \mathrm{~h}$ in LT every month. Associated with the differences in altitude, the orbit periods are also slightly different, that of CHAMP being roughly $93 \mathrm{~min}$ and that of Ørsted and SAC-C being roughly $100 \mathrm{~min}$.

This implies that even during times when CHAMP and Ørsted cover nearly the same local time regions, they will be at opposite locations in their orbits roughly once per day, i.e. part of each day they will be over the same polar region at roughly the same time and we can use this to make consistency checks of their measurements of the ionospheric currents. During other parts of the day, they will provide near-simultaneous measurements for both the northern and southern polar regions and this allows us to investigate conjugacy issues of the ionospheric currents. There is, however, a caveat in this. Due to the displacement of the magnetic poles from the geographic poles, there is a daily variation in how polar, relative to the magnetic pole, each orbit appears. This variation is much larger for the south pole than for the North pole, and also is much larger for the Ørsted and SAC$\mathrm{C}$ satellites than for CHAMP (due to the higher inclination of the CHAMP orbit). These orbital differences imply that it is sometimes difficult to compare directly the results of polar crossings by the different satellites (either of the same polar region or of opposite polar regions). One consequence of these orbital variations is that they cause the satellite path to cut through the auroral oval not always at a near $90^{\circ}$ angle. In the worst cases, the satellite track does not cut through the oval at all, but rather skims it. This happens for a few Ørsted and SAC-C orbits daily over the southern polar region.

The satellites all deliver high precision magnetic field measurements (Olsen et al., 2000b). For the present study we have used $1 \mathrm{~s}$ measurements of the magnetic field strength averaged to $5 \mathrm{~s}$ data values.

\subsection{Current determination algorithm}

To derive from the magnetic measurements estimates of the ionospheric currents, we adopt the method introduced by Olsen (1996) with only minor modifications. In the strong Earth magnetic field, to a very good approximation, only the parallel component (to the background, internal field) contributes to the absolute value of the field, as can be seen from the following simple estimate. The absolute value of the magnetic field, $|\boldsymbol{B}|$, in terms of its components parallel, $\boldsymbol{B}_{\|}$, and perpendicular, $\boldsymbol{B}_{\perp}$, to the internal field reads:

$|\boldsymbol{B}|=\sqrt{\boldsymbol{B}_{\|}^{2}+\boldsymbol{B}_{\perp}^{2}}$.

At satellite altitude over the polar region, the internal field is of the order of $40000 \mathrm{nT}$, whereas external contributions to the field, both in the parallel and perpendicular directions, are of the order of $100 \mathrm{nT}$. Hence, it follows that:

$$
|\boldsymbol{B}|=\left|\boldsymbol{B}_{\|}\right|\left(1+O\left(\frac{\boldsymbol{B}_{\perp}^{2}}{\boldsymbol{B}_{\|}^{2}}\right)\right)=\left|\boldsymbol{B}_{\|}\right|\left(1+O\left(10^{-5}\right)\right) \text {. }
$$



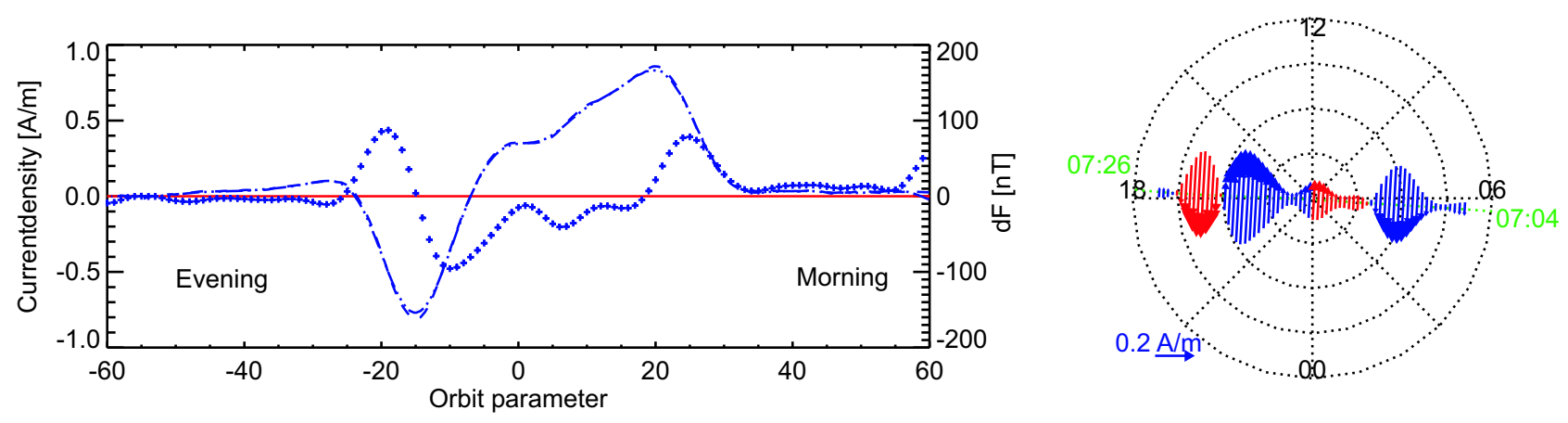

Fig. 1. Left: (Orsted orbit 9015, north) This figure illustrates the results of the current derivation technique applied to a single north polar crossing of the Ørsted satellite starting around 07:00 UT on 10 November 2000. Plotted are the measured and modeled field strength deviations (right scale and dotted and dashed curves, respectively), together with the modeled current density (crosses at the position of the individual line currents with positive currents defined as moving from right to left, as seen from the satellite flying from morning to evening). These are plotted as functions of orbit parameter. Right: On a polar map of the northern high latitude ionosphere, the derived line current densities are plotted as vectors perpendicular to the orbit track (the assumed direction of the modeled line currents). The size of each vector indicates the strength of the current density. Red color indicates mostly eastward directed vectors and blue color mostly westward directed vectors. Note, that with this definition, the color or arrows changes near the pole, while the actual direction is unchanged. The map is in Apex, MLT coordinates, with noon at the top and dusk to the left. Each latitude circle is $10^{\circ}$. The numbers in green mark the begin and end UT times for the pass.

We take advantage of this and use field strength as an approximation for the parallel component in our analysis. Due to problems in obtaining continually high precision attitude information for the field measurements, this greatly improves the accuracy and availability of measurements from the three satellite missions used here. Magnetic field strength variations are obtained by subtracting from the measured field strength value the contribution from the Earth's internal magnetic field, using the latest updated model (Olsen et al., 2000a; Olsen, 2001), as well as from a symmetric ring current, the contribution of which is estimated based on the $D_{s t}$ index.

Following Olsen (1996) we assume that only horizontal ionospheric currents (not field aligned-currents flowing into and out of the ionosphere) contribute to the parallel component of the magnetic field as measured at the satellites. The horizontal currents are modeled as a set of infinite line currents flowing perpendicular to the satellite orbit plane in a height of $115 \mathrm{~km}$ (the approximate location of the ionospheric E-region). As explained in Olsen (1996), the contributions from the set of model line currents to the set of magnetic field variation measurements along the satellite track across the polar region define a linear problem which is then solved by regularized least-squares inversion.

The model line currents are placed with a separation of $1^{\circ}$ in the ionosphere (corresponding to $111 \mathrm{~km}$ at $115 \mathrm{~km}$ altitude), but since the satellites all take measurements more than $200 \mathrm{~km}$ above the current source, their actual altitude above the currents is what determines the lower limit to the resolution of the current system structures that they can resolve. In particular, it will be apparent later when comparing the results of the CHAMP and Ørsted satellites that the results of the former are more detailed due to its closer proximity to the current source. A last remark that we should like to make about the approach adopted for this study is that we have ignored, for now, the effects of electromagnetic induction in the Earth's interior. These effects will change the absolute value of the modeled currents in the ionosphere, but will not change much their shape and location.

An example of the results of applying this technique to measurements of the Ørsted satellite is illustrated in Fig. 1. The magnetic field variations measured (dotted curve) along the satellite track during a single north polar crossing (going from morning side to dusk side, right to left in the figure) are displayed in the left panel. The intensity of the model line currents as they are distributed along the track are indicated with crosses. The corresponding (modeled) values for the magnetic field strength (dashed curve) are also plotted and the fact that this curve is virtually indistinguishable from the curve of the measured values demonstrates the accuracy of the model fit. These are all plotted as functions of the orbit parameter, defined as the angular distance between a location in the orbit and the point of the orbit closest to the geographic pole (negative on the evening side and positive on the dayside). The electrojets in this case are identified as the two humps of positive currents at roughly $-20^{\circ}$ and $25^{\circ}$, respectively, for the orbit parameter. In addition, we see model currents distributed across the polar cap. These are not considered completely realistic, since we do not expect the approximation of cross track infinite line currents to be as good for the polar cap region as for the auroral region. The magnetic signal from which these currents are derived, however, is real and so we consider the polar cap model currents to partly represent real currents in this area and partly represent the long distance effect to the magnetic field in the polar cap from auroral current systems. A different view of the model currents is presented in the right panel, which shows the satellite track on a polar map of the northern high-latitude ionosphere. In 
this plot, we identify the WAEJ with blue arrows at roughly $70^{\circ}$ magnetic latitude on the morning side and the EAEJ with red arrows located at roughly $65^{\circ}$ on the evening side. The presence of a strong westward current just poleward of the EAEJ is also clear in this representation of the currents. This is the result of a large negative $Y$-component in the IMF at this time, causing a large, round convection cell in the dawn side of the northern polar ionosphere.

\subsection{Satellite current indices}

From the model currents derived for each polar crossing, we define a set of indices as measures of the electrojet currents. One index is defined as the sum of the (mostly) westward directed currents, each weighted by a factor given as $\sin \left(\theta_{m}\right)$, where $\theta_{m}$ is magnetic co-latitude, to put emphasis on the auroral currents (lower latitude) rather than on the polar cap currents. The unit for the index is MA $\left(10^{6} \mathrm{~A}\right)$. This index is related to the ground-based $A_{L}$ index. Similarly, a second index is defined as the weighted sum of the (mostly) eastward directed currents and is related to the ground $A_{U}$ index. Finally, a third index is defined simply as the sum of the two previous indices and this index is related to the ground $A_{E}$ index.

\section{Results}

\subsection{The current indices}

The electrojet activity has been monitored since the early sixties by the set of geomagnetic indices: $A_{L}, A_{U}$, and $A_{E}$, defined by Davis and Sugiura (1966). These are based on the magnetic variations measured at 12 ground-based magnetometer stations located in the northern polar region at standard (average) auroral latitudes. The $A_{E}$ indices provide a simple and well proven means of monitoring the auroral electrojet activity (e.g. Baker, 1986). As was discussed right from the beginning, however, they are flawed with obvious problems related to the limited coverage of the AEstations both longitudinal and latitudinal (e.g. Davis and Sugiura, 1966; Rostoker, 1972; Kamide and Akasofu, 1983; Ahn et al., 2000). Nevertheless, they continue to play a large role as a quantitative parameter describing various aspects of the solar-terrestrial environment (e.g. Weimer et al., 1990; Lu et al., 1998), and the $A_{E}$ index is recognized as a metric for space weather modeling and forecasting (e.g. Vassiliadis et al., 1993; Gleisner and Lundstedt, 1997; NSWP, 2000).

Recent work has often adopted extended definitions of the indices, utilizing all existing magnetic measurements in the auroral regions (between 55 and 76 degrees magnetic latitude), to improve the coverage of the electrojets (Kamide et al., 1981; Shue and Kamide, 1998; Lu et al., 1998). This is illustrated in Fig. 2, which shows the distribution of Northern Hemisphere stations with magnetic latitude greater than $50^{\circ}$, that provide data more or less routinely at this time.

As a first example to demonstrate the overall good validity of the satellite derived currents, we compare the derived

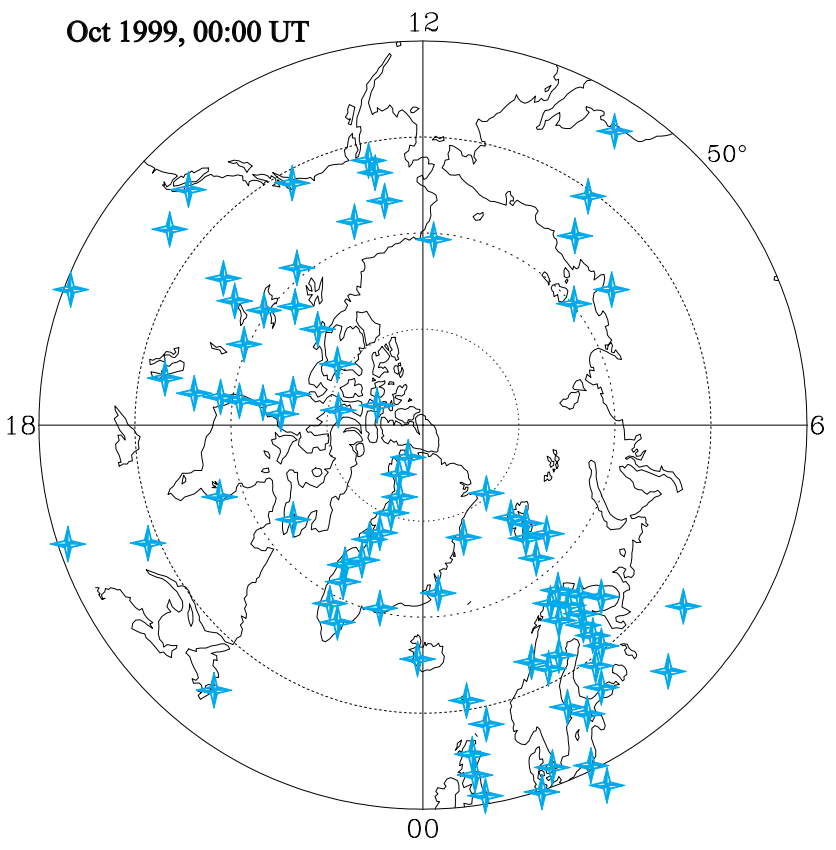

Fig. 2. A map showing the distribution of ground magnetometer stations in the northern polar region from which data is typically available at this time (the map is specifically of stations providing data in October 1999). The map is in Apex (Richmond, 1995) magnetic latitude and magnetic local time (MLT) coordinates. Noon MLT is at the top and the distribution is shown at 24:00 UT.

current indices with extended $A_{L}, A_{U}$, and $A_{E}$ indices for the storm period 9-12 November 2000. This storm has been modeled with the AMIE technique (Richmond, 1992) and to this end, ground magnetic measurements from all available stations in the polar regions were collected and processed. These include 69 auroral stations, most of which are located in the Northern Hemisphere (cf. Fig. 2). These measurements provide the ground-based indices that we use in this comparison. For this period we have data from two of the satellites: CHAMP and Ørsted, and they are in almost identical orbit planes. The orbit of CHAMP crosses the equator at roughly 04:00 and 16:00 MLT and that of Ørsted at roughly 05:00 and 17:00 MLT. Both satellites are northward bound on the morning side of the orbits.

The indices for Northern and Southern Hemisphere polar crossings are illustrated in the upper and lover panels, respectively, of Fig. 3. Full lines represent the ground-based indices, full circles represent the Ørsted current indices, and open circles represent the CHAMP current indices. The correlation coefficients quoted in the figure are calculated by combining all entries for a given index from the two satellites and correlating the resulting single time series with the corresponding ground index, for which 25-min averages interpolated to the times of the satellite indices are used. For the Northern Hemisphere the ground-based and satellite indices clearly are very highly correlated, reaching a correlation coefficient between $A_{E}$ and the total current index of 0.91. The correlations for the Southern Hemisphere are less 

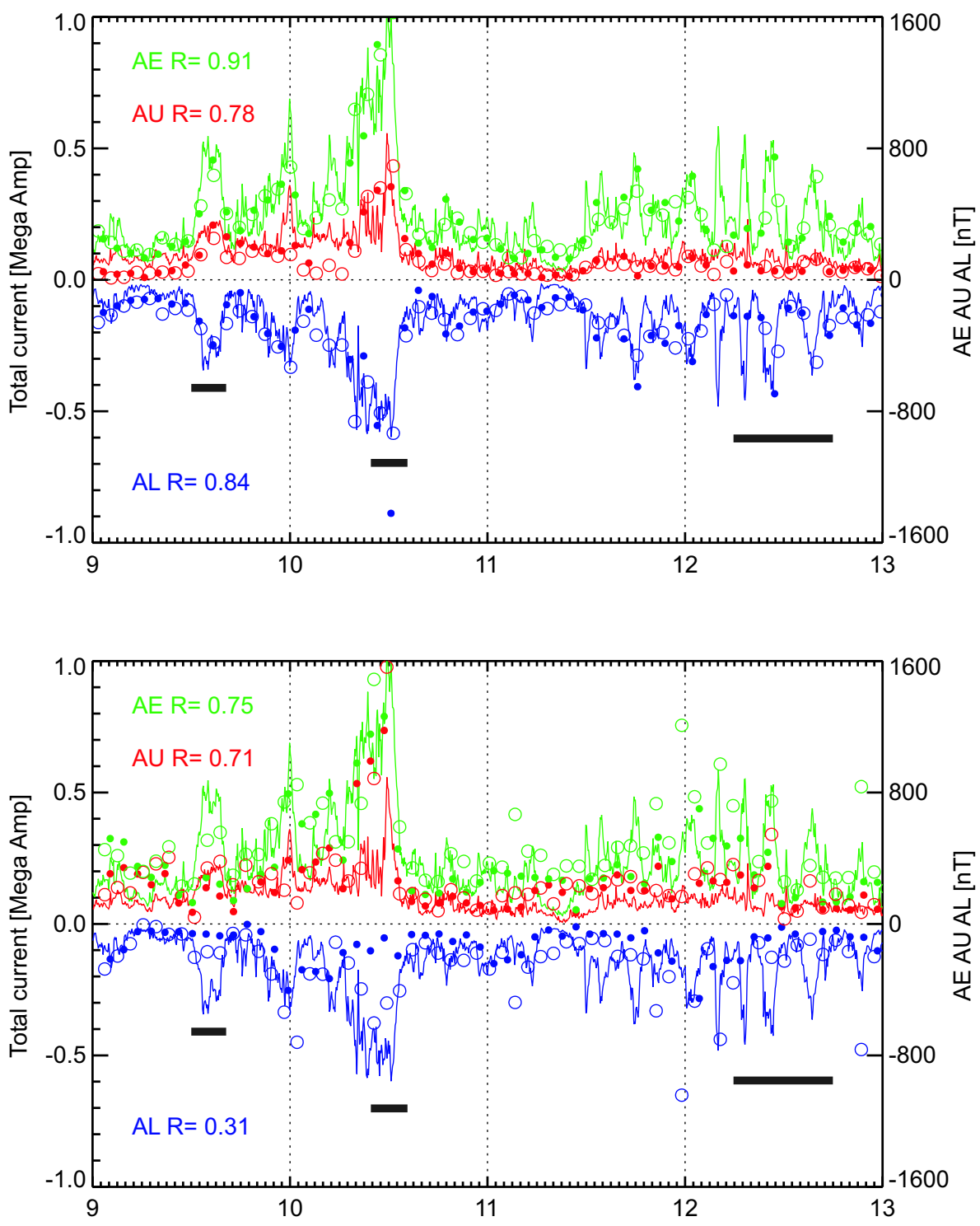

Fig. 3. The time series of ground-based auroral indices and current indices from both Ørsted and CHAMP for the time period 9-12 November 2000 are displayed. The upper panel is for the Northern Hemisphere, the lower panel for the Southern Hemisphere. In both panels, the $A_{L}$ index and the corresponding satellite indices for westward current are drawn in blue color; the full line is the 5-min average $A_{L}$ index, the filled circles are Ørsted values, and the bigger open circles are CHAMP values. The $A_{U}$ index and the corresponding eastward satellite current indices are plotted in the same way in red color. Finally, in the green color the $A_{E}$ index is plotted, together with the total absolute current indices for the two satellites. Black bars in the upper panel point out periods that are discussed specifically in the text.

convincing, in particular for the $A_{L}$ index. The fact that the southern polar crossings more often pass more often rather far from the magnetic pole than the northern ones and hence, traverse the auroral currents at an oblique angle, might account for some of this. However, it also seem very clear at times that real differences between the currents in the two hemispheres exist (examples hereof will be discussed later). Therefore, our result for the Southern Hemisphere, at least partly, also simply illustrates the fact that the ground-based indices are, inherently, mostly Northern Hemisphere indices.

A specific difference between the satellite indices and the ground indices is that the satellite indices constantly monitor the currents in a given set of local time regions (approx- imate $12 \mathrm{~h}$ apart), whereas the ground indices are designed to cover, more or less, all local times. It is well known that, generally, the strongest contribution to the $A_{L}$ index is in the early morning hours, around 03:00 MLT, and the main contribution to the $A_{U}$ index is in the dusk region (around 18:00 MLT) (e.g. Kauristie et al., 1996; Ahn et al., 2000). Consequently, one might think that the orbit of the satellites for the results shown here is particularly well suited for obtaining good correlation with the ground indices. We have tested this with Ørsted data for two other storm periods for which the comprehensive set of magnetometer data has been collected to run the AMIE procedure. For 13-16 July 2000, the orbit was similar to the case presented here, crossing the 
Equator at roughly 06:00 and 18:00 MLT and for the Northern Hemisphere, a correlation with $A_{E}$ of 0.95 was found. For the period 10-14 October 1999, however, the satellite was in a near noon-midnight orbit, crossing the Equator at roughly 11:00 and 23:00 MLT, and, even so, a correlation with $A_{E}$ of 0.90 was found. This result is somewhat surprising, showing that the local time coverage does not seem to be as important as one might have expected. While we are not yet able to fully explain this result, it has likely to do with the fact that the satellites are relatively high above the current source. This means that their measurements are also influenced by the remote effect of current systems quite some distance away from the satellite ground track. Part of the current derived for the polar cap region, for example, we attribute to such effects. Another inherent difference between the two types of indices is that the satellite indices include a measure of the widths of the electrojet currents (being defined as integrals), whereas the ground indices are defined solely to monitor the maximum intensities of the currents.

To illustrate some further features of the satellite results, we now present a detailed look at a few selected time periods indicated by black bars in Fig. 3. Sometimes (recurrent approximately once a day), the two satellites are far from each other in their respective orbits, i.e. crossing the poles at significantly different times (up to $50 \mathrm{~min}$ apart). For the days shown in Fig. 3, this happens in the hours around 05:00 UT. This, as well as occasionally simply filling out data gaps for each other, leads to improved time resolution of the satellite indices. It can be seen at these times that the indices from the two satellites complement each other in tracking the ground based indices' curves. An illustrative example is the tracking of the activity in the hours before and around 12:00 UT on 12 November 2000 (marked by the rightmost of the black bars).

Similarly, at other times, around 17:00 UT of each day in Fig. 3, the two satellites cross the same pole nearly simultaneously. At these times we can compare the results from the two satellites. Overall, we find a very good match (many open and closed circles on top of each other in Fig. 3 around these times). An illustrative example is the period just after 12:00 UT on 9 November 2000 (the leftmost black bar in Fig. 3). Maps of the actual model currents derived from the measurements of the two satellites during this time are displayed in the upper two rows of Fig. 4. Each row in Fig. 4 displays the model results of one Ørsted orbit and one CHAMP orbit, the first map showing the Ørsted northern polar crossing, the second map showing the near-simultaneous CHAMP northern crossing, and the last two maps showing the consecutive southern polar passes for the two satellites.

The first set of northern polar passes occurs around 13:00 UT on 9 November 2000. The derived current pattern is clearly consistent with the usual two-cell convection system expected to prevail for moderate auroral activity, as indicated by the (ground) indices. In particular, we observe the auroral electrojets, the EAEJ (red arrows) on the evening side at approximately $70^{\circ}$ magnetic latitude and the corresponding WAEJ (blue arrows) on the morning side at approximately $65^{\circ}$. The following set of north polar crossings occurs around 14:45 UT and shows almost the same current pattern only intensified. For both sets, the results of the two satellites are in very good agreement. In contrast, the southern passes clearly demonstrates how the satellites can miss some or all of the currents if the orbits are not close enough to the magnetic pole or pass in the wrong local time sectors.

The other example shown in Fig. 4 is from the period around 12:00 UT on 10 November 2000 (the middle black bar in Fig. 3). This is during the most intense part of this storm period. Also in this case, it is seen that the current systems for the northern passes of the two satellites agree quite well. The current profiles from the CHAMP data show more smaller scale structure, reflecting the lower latitude of this satellite but otherwise the intensity and location of the current systems are compatible with the ones observed with the Ørsted data. Small differences are observed though. For example, for the second set of passes (bottom row of Fig. 4), the EAEJ (red arrows) observed by CHAMP is broader and more intense than the one observed by Ørsted. The differences are most likely explained by the slight differences in local times and UT times between the passes of the two satellites. As in the first example, the Southern Hemisphere passes are not very polar at this time and are displaced such that they see mainly dayside currents. This makes comparisons of these profiles with the Northern Hemisphere ones or even amongst themselves not very meaningful.

\subsection{North and south current systems}

The results of the previous section were inconclusive regarding the southern polar current system due to difficulties with timing (at the interesting times the satellites were over the same pole rather than opposite poles) and orbit orientation (Ørsted crossing far from the pole on the Southern Hemisphere). It is possible, though, to find situations that allow for detailed comparisons between the north and south polar regions. We shall now present the results of such an investigation. During the days of 22-23 April 2001, the interplanetary magnetic field (IMF), as measured by the ACE spacecraft, was unusually steady, changing only very slowly over the course of these two days. Since this greatly reduces the effects of rapid dynamic changes to the ionospheric current systems, this interval provides an excellent opportunity to investigate these with our method. At this time, the CHAMP and Ørsted orbit planes, again, are close together, both being in near noon-midnight orbits. The orbit of CHAMP crosses the Equator at roughly 01:00 and 13:00 MLT and that of Ørsted at roughly 02:00 and 14:00 MLT. CHAMP is northward bound on the dayside and Ørsted on the nightside.

During the period 05:00-11:00 UT on 22 April 2001, the satellites are near opposite to each other in their orbits, i.e. they make opposite polar crossings at nearly the same time. Ionospheric current maps for eight consecutive nearsimultaneous north and south passes are displayed in Fig. 5.

The format of the maps is the same as in Fig. 4. The first pair of passes (left column, first row) occurs around 05:00 UT and the last pair (right column, last row) just before 

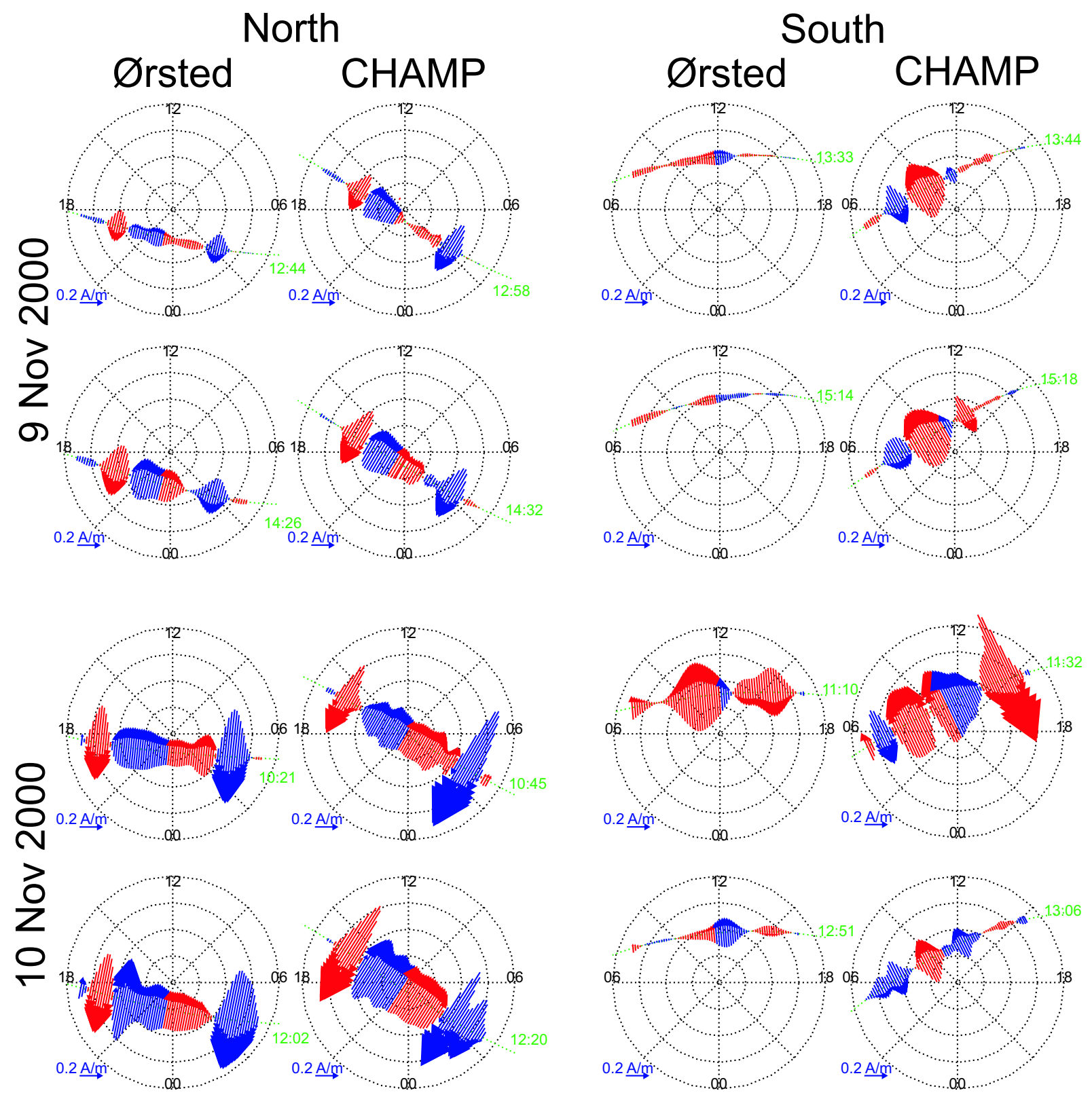

Fig. 4. Shown here are the derived currents for a few selected orbits for which the Ørsted and CHAMP satellites passed the same polar regions nearly simultaneously. The format of the polar maps is the same as in Fig. 1. The Southern Hemisphere maps are viewed as looking down on the south pole from below, i.e. noon is at the top and dusk is to the right. The start time of the passes is marked in green. Each row of maps shows from left to right a Ørsted northern pass, the corresponding (near simultaneous) CHAMP northern pass, the following Ørsted southern pass, and the corresponding CHAMP southern pass. The upper two rows show two successive orbit pairs, and likewise for the lower two rows.

11:00 UT. Values of the IMF from the ACE spacecraft for a period of $8 \mathrm{~h}$ surrounding this time interval are displayed in Fig. 6.

ACE is located $223 R_{E}$ upstream of the Earth and the solar wind speed is fairly constant throughout this period at roughly $370 \mathrm{~km} / \mathrm{s}$ (data not shown). This gives an estimated propagation time of the solar wind from the spacecraft to the Earth's magnetopause of roughly $60 \mathrm{~min}$. This delay has been included in the times shown for the measurements in Fig. 6.
It is seen in Fig. 6 that after an initial change from near zero to negative of the $Z$-component half an hour into the interval, the values of the IMF components are all fairly constant throughout the interval. The dominating components are the $Y$ - and $Z$-components, both of which are negative and at near equal value of roughly $-10 \mathrm{nT}$. This sets the stage for interpreting the observations presented in Fig. 5. Looking first at the sequence of northern polar maps, we note that they display a fairly steady and rather low intensity current system. The EAEJ at just below $70^{\circ}$ magnetic latitude on the 

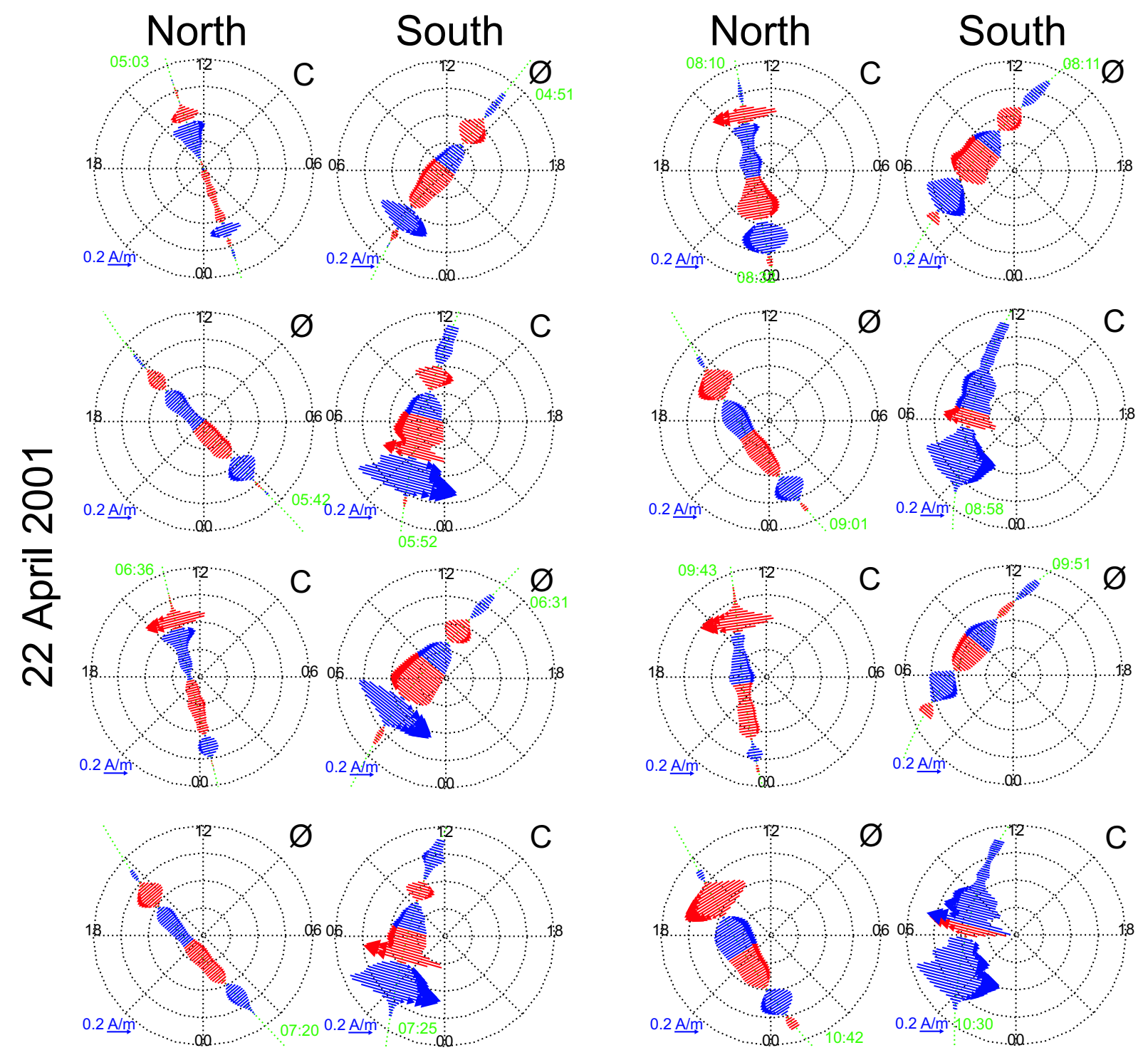

Fig. 5. The ionospheric maps of the derived currents for a sequence of orbits for which Ørsted and CHAMP passed opposite poles nearly simultaneously are shown here. The format of the polar maps is the same as in Fig. 1. In the upper right corner of each map is indicated which satellite produced the map, $\varnothing$ for Ørsted and C for CHAMP. Successive pairs of near-simultaneous north and south passes are displayed in two columns (of two maps each), starting at the top of the left column, going down through the left column, and then continuing from top to bottom through the right column. In each pair, the northern pass is to the left and the southern pass to the right, as indicated at the top of the columns.

dayside seem to intensify slowly but steadily throughout the time interval. In contrast, the WAEJ, at roughly $65^{\circ}$ magnetic latitude on the nightside, is slightly intensified in the map around 08:20 UT (right column, top), probably due to a small substorm, but otherwise remains small throughout. For the orbits that pass close to the cusp region, e.g. the third one around 06:45 UT, we observe an intensified westward current on the dayside around $75^{\circ}$ magnetic latitude just above (and related to) the EAEJ observed here. This, we believe, is the signature of the DPY current (e.g. Friis-Christensen and Wilhjelm, 1975; Friis-Christensen et al., 1985) associated with the large negative value of the $Y$-component of the IMF.
The Southern Hemisphere maps give a somewhat different picture. They too show a fairly constant current profile, though the maps towards the end of the sequence are somewhat inconclusive due to the fact that the passes become more and more displaced from the pole. On the dayside, at least for the first 5 passes which go across the noon meridian on the dayside, we observe an eastward current in the cusp region consistent with the DPY current expected for the Southern hemisphere. A variable, rather strong WAEJ is observed in the early morning region between 60 and $70^{\circ}$ magnetic latitude. It is evident that the WAEJ in this region throughout the time interval is much more intense than its counterpart in the Northern Hemisphere. This result is clearly very 


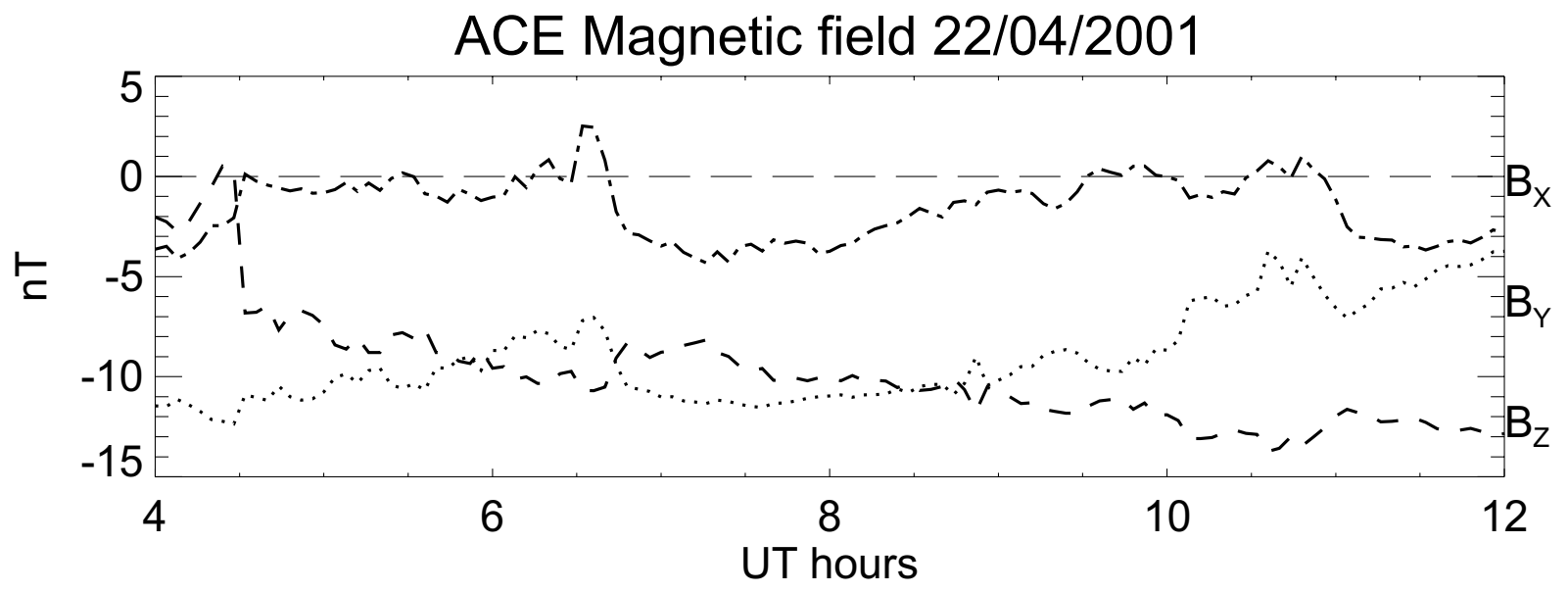

Fig. 6. The 4-min averaged measurements of the interplanetary magnetic field from the ACE spacecraft are displayed. Components, $B_{X}$ (dash-dotted line), $B_{Y}$ (dotted line), and $B_{Z}$ (dashed line), in the GSM coordinate system are shown. A delay of $1 \mathrm{~h}$ is included in the time shown for the measurements to account for the solar wind propagation from the spacecraft to the magnetopause.

robust, appearing repetitively through many passes of varying timing and likeness with their Northern Hemisphere partners. Unfortunately, we do not have similar observations for the pre-midnight region to examine whether a corresponding asymmetry exists for the EAEJ in that region.

Even so, the result is interesting. To our knowledge, this is the first study providing a direct inter-hemispheric comparison of the current systems and only a few studies exist, with the aim of direct comparisons of the electric potential or convection patterns. Lu et al. (1994) used the AMIE technique to conduct an inter-hemispheric comparison of the convection patterns during IMF conditions very similar to those for this event. For negative $Z$ - and $Y$-components of the IMF, they concluded that the convection patterns for the Northern and Southern Hemispheres were largely mirror images of each other (round-shaped dawn convection cell and crescentshaped dusk cell in the Northern Hemisphere matched by a crescent-shaped dawn cell and a round-shaped dusk cell in the Southern Hemisphere) and the cross-polar-cap potential drop is essentially the same in the two hemispheres, even when the negative $Y$-component is much larger than the $Z$ component. This confirmed earlier statistical work (e.g. Heppner and Maynard, 1987), as well as model predictions (e.g., Reiff and Luhmann, 1986; Crooker, 1988), that the convection pattern for the Southern Hemisphere during the negative $Y$-component of the IMF is the same as that of the Northern Hemisphere during the positive $Y$-component of the IMF, except possibly for seasonal effects (e.g. Crooker and Rich, 1993). Naturally, ionospheric currents have a much stronger, direct seasonal dependency as compared to convection (or electric field). The event here, however, occurs close to Equinox and more towards the northern rather than the southern summer. Furthermore, the main difference is seen in the current strength on the nightside. Hence, the observed asymmetry cannot be explained as a seasonal effect. The work of Friis-Christensen and Wilhjelm (1975) also concerned ionospheric current patterns, though only in the Northern Hemisphere, and they concluded that the IMF $Y$-component did not seem to have any substantial effect on the nightside currents. Comparing to their Fig. 3, we see no differences between the negative and positive $Y$-component cases that could account for the asymmetry observed here. It seems, therefore, that it is a real inter-hemispheric asymmetry in the currents, possibly related in some way to the complicated morphology of the tail magnetosphere during strong $Y$-components of the IMF. We speculate that this might be able to produce, for example, a difference in the particle precipitation patterns, which determine the nightside conductivity levels between the two hemispheres. A similar conclusion was reached by Tanaka (2001), who used numerical simulations to investigate asymmetries of the ionospheric convection and current systems as a function of the IMF $Y$ component. The investigation of more events during different constellations of the satellites, and also including strongly positive $Y$-component cases, as more data become available in the future, should help solve this puzzle.

\subsection{Wide local time coverage}

Even with more than 2 years of data from Ørsted, 9 months of data from CHAMP, and 6 months of data from SAC-C, it proved surprisingly difficult to find periods well suited for combined studies like the one presented above. In particular, we are interested in periods where the satellite orbits are widely separated in local time. Since SAC-C started to provide data at the end of March 2001, the satellite orbits stayed close together until June 2001. As noted in the section above, large seasonal differences in the current systems makes it difficult to study other differences during summer and winter times. Nevertheless, as a first demonstration of the capabilities of the combined data set, we show here the results for a time interval on 18 June 2001. For this day, observations were available from all three satellites, and the IMF, at least partly, fulfilled the requirements of varying slowly and having a large $Y$-component. Orientation of the orbit planes for 


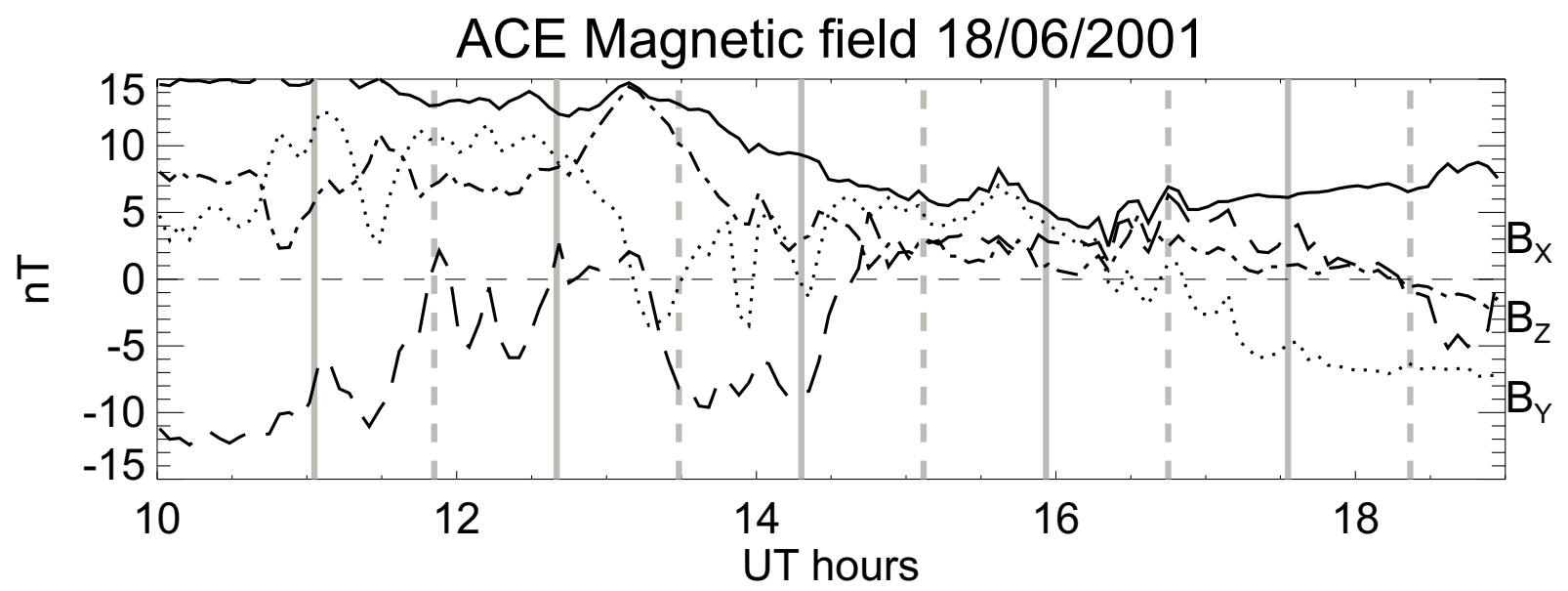

Fig. 7. The 4 min averaged measurements of the interplanetary magnetic field from the ACE spacecraft are displayed. Components, $B_{X}$ (dash-dotted line), $B_{Y}$ (dotted line), and $B_{Z}$ (dashed line), in the GSM coordinate system are shown. A delay of 65 min is included in the time shown for the measurements to account for the solar wind propagation from the spacecraft to the magnetopause. Times corresponding to each of the polar crossings displayed in Fig. 8 are indicated by the gray vertical lines; full lines mark northern crossings and dashed lines southern crossings.

the three satellites is as follows: CHAMP crosses the Equator at roughly 08:00 and 20:00 MLT; Ørsted at 02:00 and 14:00 MLT; and SAC-C at 10:00 and 22:00 MLT. The timing of the orbits is such that during the period 11:00-18:00 UT the satellites all cross the same poles nearly simultaneously.

To guide the interpretation of the derived current patterns, values of the IMF from the ACE spacecraft for a period of $8 \mathrm{~h}$ surrounding this time interval are displayed in Fig. 7. ACE is located $244 R_{E}$ upstream of the Earth and the solar wind speed is fairly constant throughout this period at roughly $375 \mathrm{~km} / \mathrm{s}$ (data not shown). This gives an estimated propagation time of the solar wind from the spacecraft to the Earth's magnetopause of roughly $65 \mathrm{~min}$. This delay has been included in the times shown for the measurements in Fig. 7. The resulting current maps for this period, covering five consecutive orbits, are displayed in Fig. 8.

The format of the polar maps is the same as in the previous figures, except that this time we plot all three nearsimultaneous passes on the same map. In general, we find good compatibility between the results from the three satellites and also observe how they complement each other in providing good coverage. We see the indications of some very interesting current systems and their evolution, in particular in the Northern Hemisphere. To assist us in relating these to the IMF, we have indicated (by the vertical bars) in Fig. 7 the times corresponding to each of the polar crossings. In estimating these times we have included a 15-min delay between the time a certain IMF value is at the magnetopause and the time of the polar crossing. This is to account for the response time of the ionosphere $(5-10 \mathrm{~min})$ and the time to set up a new current system (10-15 min) (e.g. Ridley et al., 1998). For example, the first north polar crossing occurs at 11:18 UT (taking the average between the start times for the three satellites and adding $13 \mathrm{~min}$ ) and so the time marked for the crossing in Fig. 7 is at 11:03 UT. We note that if the
IMF changes greatly on a time scale, which is not considerably longer than $15 \mathrm{~min}$, around the times of the crossings, this will affect our ability to make the connection between IMF value and current pattern.

The first Northern Hemisphere map is obtained at a time when the IMF has a steady large negative $Z$-component of roughly $-10 \mathrm{nT}$ and a comparably large positive $Y$ component. The observed current pattern reflects this primarily very well. Strong eastward currents are observed all across the dayside and into the evening region, and this agrees well with the presence of a large round convection cell on the dusk side as expected (for the Northern Nemisphere) for this IMF condition. They dominate the pattern more than expected though, leaving only a small hint of the corresponding crescent-shaped cell on the dawn side in the form of the small westward current observed around 70 ${ }^{\circ}$, 09:00 MLT. Though the IMF has become more variable by the time of the subsequent Southern Hemisphere map, the current pattern here is still consistent with the general pattern expected for the IMF with negative $Z$ and positive $Y$-components (round cell in the dawn side and crescent cell in the dusk side for the Southern hemisphere). Overall, the strengths of the currents are much smaller than for the Northern Hemisphere by a factor of 2-3. This is in line with earlier results on the differences between the summer and winter hemispheres (e.g. Friis-Christensen and Wilhjelm, 1975). We note, however, that the difference is relatively much larger for the nightside currents (comparing the currents at midnight and in the early morning region in the Southern Hemisphere with the corresponding currents at midnight and in the evening region in the Northern Hemisphere) than for the dayside currents. We are not able to explain this at present.

Continuing on to the second pair of maps in the sequence, the northern map is at a time when the $Z$-component is greatly reduced and has been so for a while. The effect of 
this is seen mainly as a general reduction in the strengths of the currents on the dusk side, together with a contraction of the round cell. In contrast, the westward current defining the crescent cell on the dawn side has intensified, leaving the resulting pattern to be in good agreement with the expectations for an IMF dominated by a positive $Y$-component. The second Southern map is obtained close to large changes in both the $Y$ - and $Z$-components. Therefore, it is somewhat surprising to find that the observed current pattern is not very different from the first one.

The third Northern Hemisphere pattern also holds a small surprise. This pattern is for a time when the IMF $Z$ component has again become large and negative, while the $Y$-component is small and variable. We see the effect of the large negative $Z$-component as a general increase again in all the currents. Most noticeable this time is the occurrence of a large westward electrojet at dawn. This is not the effect of a substorm as is evident from the small westward electrojet current observed (at almost exactly the same time) at midnight. It is curious that the current system continues to exhibit the shape of a round dusk cell and a crescent dawn cell. It would seem impossible that it could still at this time be influenced by conditions of the positive $Y$-component. This is another interesting feature of this sequence of current patterns that we will have to leave as unexplained for the time being.

The third Southern Hemisphere map and the fourth northern map occur during fairly steady and very similar IMF conditions, namely a dominant positive $Y$-component of approximately $5 \mathrm{nT}$ and a smaller positive $Z$-component of approximately $3 \mathrm{nT}$. This results in the most compatible set of maps of the sequence. Both maps again show the features of a positive $Y$-component driven system, which is approximately mirror symmetric between the two hemispheres. A small difference is observed in the crescent cell (dawn side in the Northern Hemisphere and dusk side in the southern), which is more sharply defined in the northern map (dawn side) than in the southern. Another difference is in the strength of the eastward electrojet observed in the evening side of the northern map and its corresponding (mirror symmetric) westward electrojet in the early morning region of the southern map. Given that the dayside and dawn/dusk (north/south) currents are of compatible strengths and that one would expect any differences in the currents due to differences in conductivity between the summer and the winter hemispheres to be greatest on the dayside, we speculate that this is another indication of real inter-hemispheric differences in the current patterns for this IMF condition.

At the time of the fourth southern map, the IMF $Z$ component is still positive at a slightly increased value of roughly $5 \mathrm{nT}$, whereas the $Y$-component has decreased to near 0 . This agrees well with the practically complete absence of currents observed in this map.

Concluding the sequence, the last northern and southern maps again occur during very similar IMF conditions. The IMF $Z$-component is still positive and small, between 3 and $0 \mathrm{nT}$, whereas the $Y$-component is the dominant component

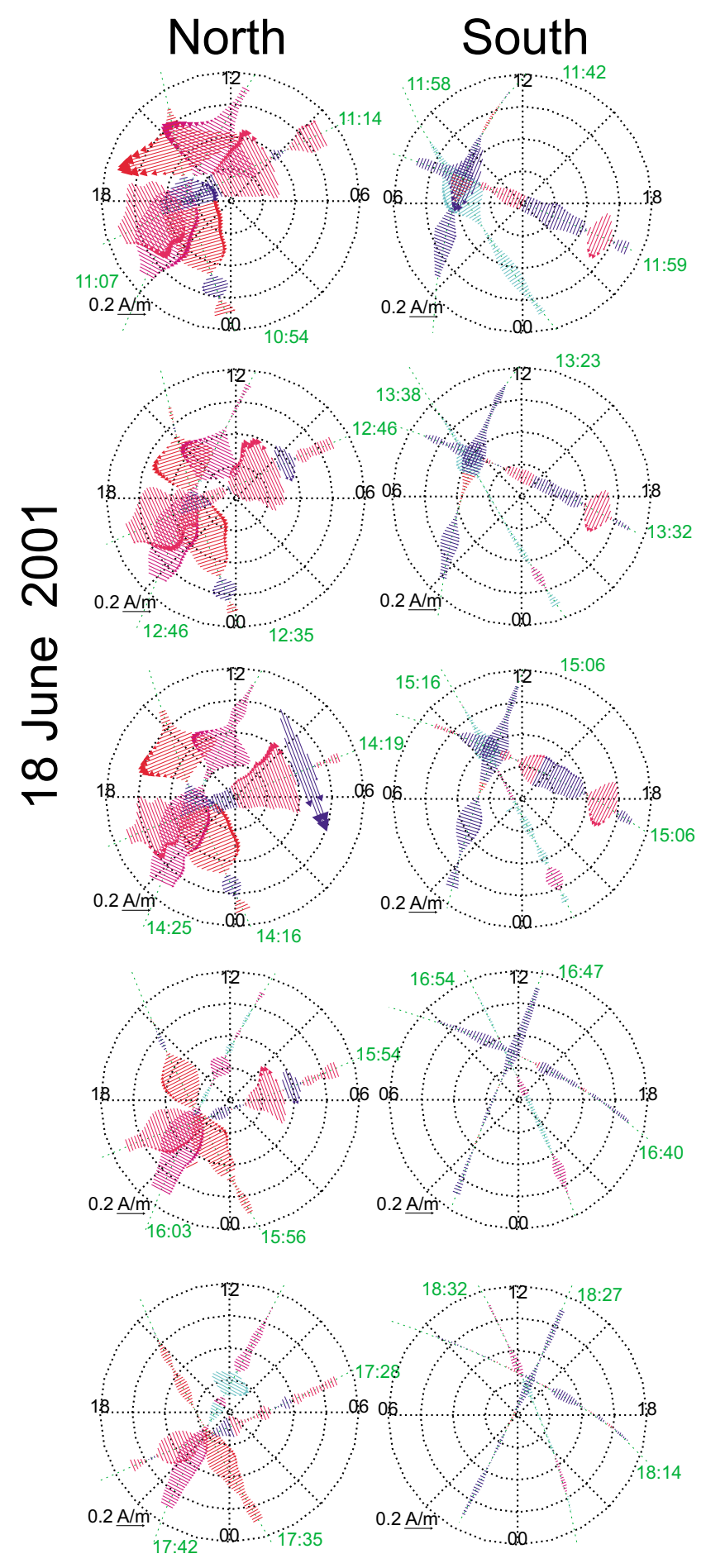

Fig. 8. Displayed here are the combined ionospheric current maps for 5 consecutive orbits on 18 June 2001. The format of the polar maps is like the one in Fig. 1. Model currents from all three satellites, Ørsted, CHAMP and SAC-C are overlaid in the plots. Ørsted currents are plotted in blue and red (same as previous figures), CHAMP currents in purple and crimson, and SACC currents in light blue and magenta. Start times for the passes are marked in green. The Northern Hemisphere crossings are to the left and the Southern Hemisphere crossings to the right, as marked at the top. 
at a value of roughly $-6 \mathrm{nT}$. This means that the polarity of the IMF $Y$-component seems to be the only difference between the solar wind conditions defining this set of maps and those of the third southern, fourth northern pair of maps described above. Consequently, we would expect these two sets of maps to be mirror images of each other. We note that the solar wind dynamic pressure (data not shown) exhibits a slow steady decrease of less than $30 \%$ (from roughly $11 \mathrm{nPa}$ to roughly $8 \mathrm{nPa}$ ) over the time interval from 15:00 UT to 19:00 UT and thus cannot be the source of any major differences between the two sets. As is evident from Fig. 8, however, the observations do not match this expectation either for the northern or southern maps.

For the northern maps, we do see the change in the sign of the current in the cusp region, around $80^{\circ}, 12: 00$ MLT, from eastward in the fourth map to westward in the last map, indicative of a change from positive to negative IMF $Y$-component. Apart from this, however, the current pattern in the last map is mainly the same as in the fourth map, only with much reduced amplitudes, particularly on the dayside. Returning to Fig. 7, we note that the main change from positive to near zero for the IMF $Y$-component occurred more than one hour prior to the time marked for the last northern map. Further, the main change towards a large negative value for the $Y$-component occurred more than 20 min prior to this time. The implication hereof is that the total reaction time for the ionosphere to set up the new current system in response to the IMF change was 35-75 min (taking into account the 15 min that we had already included in the marking of the crossing). This is extremely long and does not seem consistent with previous results (see discussion in Ridley et al., 1998). We have not been able to identify any unusual feature about the event presented here, which could explain this result.

Just as puzzling is the result for the southern maps. While the current system observed in the third map was found to match the corresponding northern current system very well, this is not so for the last southern map. Like the preceding southern map, a complete absence of currents is observed also in this map and, thus, it does not match its northern counterpart (last northern map), nor does it match the third southern map. It seems rather unlikely that this reflects a real difference in how the winter (Southern) Hemisphere reacts to the positive and negative IMF $Y$-components, respectively, since no such effect has been observed in earlier studies (e.g. Friis-Christensen and Wilhjelm, 1975). A more likely possibility, in our opinion, is that it has to do with a difference in preconditioning of the magnetosphere at the times of the two maps. The third map occurred after an interval of about 45 min of northward IMF (cf. Fig. 7, again taking into account the 15 min delay time included in the marks for the crossings), whereas the last map is preceded by more than $3 \mathrm{~h}$ of northward IMF. The mechanism by which this kind of preconditioning could influence the current systems in the observed way, however, is not clear.

In conclusion, we seem to be left with a number of unresolved questions when trying to explain the observed se- quence of current maps in both hemispheres, based on the observed conditions in the solar wind.

\section{Conclusions and outlook}

We have demonstrated some of the ways in which geomagnetic measurements from low altitude polar orbiting satellites can contribute to research into the auroral electrojet current systems, as well as the monitoring hereof. Observations from three current satellite missions, CHAMP, Ørsted, and SAC$\mathrm{C}$ are used. The very basic measurement of magnetic field intensity along the orbit track is utilized here. These measurements are much more readily available, are accurate and stable, and have much fewer problems with data gaps than the full vector measurement data set that, otherwise, is the primary product of these missions.

A set of current indices were derived based on these measurements and it was shown that these replicate the profiles of the traditional electrojet indices, $A_{L}, A_{U}$, and $A_{E}$, to an astounding degree. This can be seen as the satellite-based version of the results found by Kauristie et al. (1996), who showed that for a single meridional chain of ground magnetometers in certain local time sectors (around 02:00 MLT for $A_{L}$ and 20:00 MLT for $A_{E}$ ), the local indices measured electrojet activity as well or better than the traditional (12 station) global indices. In comparison, the satellite-based observations have a great potential for contributing additional information. First, the satellites stay continually in the same local time region (only slowly changing over the cause of days or months for CHAMP and Ørsted) or can cover several local time regions simultaneously (combining all 3 satellites). Second, they also hold the potential for contributing information about the latitudinal location and width of the electrojet currents. The satellite indice, defined as integral indices, already include some extra information as compared to the groundbased indices, but beyond this, we have not yet explored this possibility much further. To do so, we first would need to devise a method by which to model the derived current profiles in order to define and extract the interesting parameters, e.g. latitude and width.

Most importantly, with this method and data set, we cover the Southern as well as the Northern Hemisphere. Exciting preliminary results were obtained on the inter-hemispheric differences in the ionospheric current systems. While the interpretation remains somewhat inconclusive at this point, the results do point to some of the outstanding problems in our understanding of the ionospheric electrojet currents, as well as the potential of this combined data resource to address such questions.

Acknowledgements. The CHAMP satellite project is supported by grants from the German Space Agency (DLR) under contract FKZ 50 EP 9587. The Ørsted Project was made possible by extensive support from the Ministry of Trade and Industry, the Ministry of Research and Information Technology, and the Ministry of Transport in Denmark. The ground magnetometer data used in this study were provided by T. Hughes at Canadian Space Agency, L. Hakki- 
nen at Finnish Meteorological Institute, J. Watermann at Danish Meteorological Institute, L. Morris at National Geophysical Data Center of NOAA, K. Yumoto and the STEL at Nagoya University, G. van Beek of the Geological Survey of Canada, J. Posch at Augsburg College, D. Milling at University of York, O. Troshichev at the Arctic and Antarctic Research Institute in Rassia, K. Hayashi at University of Tokyo in Japan, A. T. Weatherwax at the University of Maryland, G. Burns at the Atmospheric and Space Physics group at Australian Antarctic Division, and M. Pinnock at the British Antarctic Survey. Work at HAO/NCAR was supported by the NASA SEC Guest Investigator Program and work at NASA GSFC was supported by the National Research Council Fellowship program.

Topical Editor M. Lester thanks K. Kauristie and another referee for their help in evaluating this paper.

\section{References}

Ahn, B.-H., Kroehl, H. W., Kamide, Y., and Kihn, E. A.: Universal time variations of the auroral electrojet indices, J. Geophys. Res., $105,267,2000$.

Baker, D. N.: Statistical analyses in the study of solar windmagnetosphere coupling, in: Solar wind-magnetosphere coupling, (Eds) Kamide, Y. and Slavin, J. A., p. 17, Terra Sci., Tokyo, 1986.

Crooker, N. U.: Mapping the merging potential from the magnetopause to the ionosphere through the dayside cusp, J. Geophys. Res., 93, 7338, 1988.

Crooker, N. U. and Rich, F. J.: Lobe-cell convection as a summer phenomenon, J. Geophys. Res., 98, 13 403, 1993.

Davis, T. N. and Sugiura, M.: Auroral electrojet activity index $A_{E}$ and its universal time variations, J. Geophys. Res., 71, 785, 1966.

Friis-Christensen, E. and Wilhjelm, J.: Polar cap currents for different directions of the interplanetary magnetic field in the $y-z$ plane, J. Geophys. Res., 80, 1248-1260, 1975.

Friis-Christensen, E., Kamide, Y., Richmond, A., and Matsushita, S.: Interplanetary magnetic field control of high-latitude electric fields and currents determined from Greenland magnetometer data, J. Geophys. Res., 90, 1325, 1985.

Gleisner, H. and Lundstedt, H.: Response of the auroral electrojets to the solar wind modeled with neural networks, J. Geophys. Res., 102, 14 269, 1997.

Heppner, J. P. and Maynard, N.: Empirical high-latitude electric field models, J. Geophys. Res., 92, 4467, 1987.

Kamide, Y. and Akasofu, S.-I.: Notes on the auroral electrojet indices, Rev. Geophys. Space Phys., 21, 1647, 1983.

Kamide, Y., Richmond, A. D., and Matsushita, S.: Estimation of ionospheric electric fields, ionospheric currents, and fieldaligned currents from ground magnetic records, J. Geophys. Res., 86, 801, 1981.

Kamide, Y., Richmond, A. D., Emery, B. A., Hutchins, C. F., Ahn, B.-H., de la Beaujardiere, O., Foster, J. C., Heelis, R. A., Kroehl, H. W., Rich, F. J., and Slavin, J. A.: Ground-based studies of ionospheric convection associated with substorm expansion, J. Geophys. Res., 99, 19451, 1994.

Kauristie, K., Pulkkinen, T. I., Pellinen, R., and Opgenoorth, H. J.: What can we tell about global auroral-electrojet activity from a single meridional magnetometer chain?, Ann. Geophysicae, 14, 1177, 1996.
Lu, G., Richmond, A., Emery, B., Reiff, P., de la Beaujardiere, O., Rich, F., Denig, W., Kroehl, H., Lyons, L., Ruohoniemi, J., FriisChristensen, E., Opgenoorth, H., et al.: Interhemispheric asymmetry of the high-latitude ionospheric convection pattern, J. Geophys. Res., 99, 6491, 1994.

Lu, G., Baker, D. N., McPherron, R. L., Farrugia, C. J., Lummerzheim, D., Ruohoniemi, J. M., Rich, F. J., Evans, D. S., Lepping, R. P., Brittnacher, M., Li, X., et al.: Global energy deposition during the January 1997 magnetic cloud event, J. Geophys. Res., 103, 11 685, 1998.

Lühr, H., Maus, S., Rother, M., and Cooke, D.: First in-situ observation of night-time F-region currents with the champ satellites, Geophys. Res. Lett., 2001.

Neubert, T., Mandea, M., von Frese, G. H. R., Primdahl, F., Jørgensen, J. L., Friis-Christensen, E., Stauning, P., Olsen, N., and Risbo, T.: Ørsted satellite captures high-precision goemagnetic field data, EOS, 82, 81, 2001.

NSWP: National Space Weather Program Implementation plan 2nd edition, FCM-P31-2000, Committee for Space Weather, Office of the Federal Coordinator for Meteorology, Washington, DC, 2000.

Olsen, N.: A new tool for determining ionospheric currents from magnetic satellite data, Geophys. Res. Lett., 23, 3635, 1996.

Olsen, N.: A model of the geomagnetic field and its secular variation for epoch 2000, Geophys. J. Int., 2001.

Olsen, N., Holme, R., Hulot, G., Sabaka, T., Neubert, T., et al., Ørsted Initial Field Model, Geophys. Res. Lett., 27, 3607, 2000a.

Olsen, N., Sabaka, T., and Tøffner-Clausen, L.: Determination of the IGRF 2000 model, Earth Planet. Space, 52, 1175, 2000b.

Reiff, P. H. and Luhmann, J. G.: Solar wind control of the polar cap voltage, in: Solar Wind-Magnetosphere Coupling, (Eds) Kamide, Y. and Slavin, J. A., p. 452, Terra. Scientific, Tokyo, 1986.

Richmond, A.: Assimilative mapping of ionospheric electrodynamics, Adv. Space Res., 12, 659, 1992.

Richmond, A. D.: Ionospheric electrodynamics using magnetic apex coordinates, J. Geomag. Geoelectr., 47, 191, 1995.

Richmond, A. D., Lu, G., Emery, B. A., and Knipp, D. J.: The amie procedure: Prospects for space weather specification and prediction, Adv. Space Res., 22, 103, 1998.

Ridley, A., Lu, G., Clauer, C., and Papitashvili, V.: A statistical study of the ionospheric convection response to changing interplanetary magnetic field conditions using the assimilative mapping of ionospheric electrodynamics technique, J. Geophys. Res., 103, 4023, 1998.

Rostoker, G.: Geomagnetic indices, Rev. Geophys., 10, 935, 1972.

Shue, J.-H. and Kamide, Y.: Effects of solar wind density on the westward electrojet, in: Substorm-4, (Eds) Kokubun, S. and Kamide, Y., p. 677, Terra Sci., Tokyo, 1998.

Tanaka, T.: Interplanetary magnetic field $B_{y}$ and auroral conductance effects on high-latitude ionospheric convection patterns, J. Geophys. Res., 106, 24 505, 2001.

Vassiliadis, D., Sharma, A. S., and Papadopoulos, K.: An imperical model relating the auroral geomagnetic activity to the interplanetary magnetic field, Geophys. Res. Lett., 20, 1731, 1993.

Weimer, D. R., Maynard, N. C., Burke, W. J., and Liebrecht, C.: Polar cap potentials and the auroral electrojet indices, Planet. Space Sci., 38, 1207, 1990. 